\title{
COMPARISON OF CONTOUR FEATURE BASED AND INTENSITY BASED INSAT-3D MET IMAGES COREGISTRATION FOR SUB PIXEL ACCURACIES
}

\author{
Subbiah Manthira Moorthi ${ }^{1}$ and Ramamoorthy Sivakumar ${ }^{2}$ \\ ${ }^{1}$ Space Applications Centre, ISRO, Gujarat, India \\ ${ }^{2}$ Department of Civil Engineering, SRM University, India
}

\begin{abstract}
Image registration in meteorological images that are acquired continuously for their use in weather forecast activities and other related scientific analysis is a critical requirement. Meteorological images are obtained from geostationary orbits in visible, infrared, water vapor channels covering a large frame of several hundreds of kilometres of geographical extent which generally involve bidirectional scanning to cover larger extents. The acquired images have to be guaranteed for their geometric fidelity to a standard of choice among themselves by image registration. Registration of such images require to deal with low contrast, cloud and snow occlusions apart from navigation data uncertainties. Nevertheless, sub pixel accuracies are demanded for image analysis and geophysical parameters derivations. Feature based registration techniques are commonly used and intensity based techniques are also put to use in these contexts rarely. The proposed feature based approach uses a land water boundary data extraction with phase correlation of image blocks and proposed the intensity based approach tackles the same problem without any preprocessing step using a sampler-metric-transform-optimizer procedure. A comparison of these two approaches is pursued here in this article using various channel data sets of INSAT-3D satellite for sub pixel accuracies.
\end{abstract}

Keywords:

Image Registration, Phase Correlation, Mutual Information, Optimization, Deformation, Transforms

\section{INSAT-3D SYSTEM}

INSAT-3D is the meteorological satellite launched by ISRO in July 2013. It has two primary sets of payloads namely the six channel IMAGER and the nineteen channel SOUNDER. The IMAGER generates images of earth and its environment in various spectral channels of meteorological importance whereas the SOUNDER provides vertical temperature and moisture profiles of the atmosphere. Kalpana-1 and INSAT-3A are other previous meteorological satellites of ISRO. INSAT-3D with its advanced payloads, is designed to cater to the current meteorological requirements of the country [1]. INSAT-3D is located at $84^{\circ}$ East longitude in a geostationary orbit. INSAT-3D data is received and processed at the Meteorological Data Utilisation Centre of IMD, New Delhi as well as at Bopal Earth Station (BES), Space Application Center, Ahemadabad.

\subsection{SYSTEM CHARACTERISTICS AND PAYLOAD OPERATIONS}

For met applications, the imaging channels are designed in visible $(0.55-0.75 \mu \mathrm{m})$ region and Infra-red region $(1-14 \mu \mathrm{m})$. The telescope focuses the visible and IR radiation which is divided into six wavelength bands by dichroic beam splitters first separating the visible and short wave infrared band from the IR channels and further dividing the IR into four spectral bands or channels. These four channels are centered at $3.9 \mu \mathrm{m}, 6.8 \mu \mathrm{m}$, $10.8 \mu \mathrm{m}$ and $12.0 \mu \mathrm{m}$. The visible signal focuses onto a silicon detector and the SWIR signal onto an Indium-Gallium-Arsenide (InGaAs) detector. The IR signals are directed to the cooled detectors through wavelength defining band-pass filters. The visible portion of the incident energy $(0.57 \mu \mathrm{m}$ to $0.75 \mu \mathrm{m})$ is directed to a staggered array of eight square silicon detectors of $85 \mu \mathrm{m}$ each, which provide a nominal IFOV of 28 micro radians per detector. This corresponds to $1 \mathrm{~km} \times 1 \mathrm{~km}$ square on earth. Similarly, the detectors for the SWIR channel are also 8-detector array to provide 28 micro radian IFOV per detector. The ground resolution at nadir is nominally $1 \mathrm{~km} \times 1 \mathrm{~km}$ for Visible and SWIR channels, $4 \mathrm{~km} \times 4 \mathrm{~km}$ for MIR, TIR-1 and TIR-2 channels and $8 \mathrm{~km} \times 8 \mathrm{~km}$ for $\mathrm{WV}$ channel [1].

The payload experiences diurnal temperature variations in the orbit due to varying position of sun. Due to sensitivity of IR measurements to temperature, in-orbit calibration of the Infra-red channels is carried out by periodic viewing of on-board blackbody and deep space during the imaging. The satellite also undergoes yaw-flip and orbit maintenance periodically during the year. As the position of the satellite is stationary with respect to earth, the payload scans the earth and its environs by means of opto-mechanical scanning. The movement of the scan mirror is facilitated in two directions by servo motor control [2]. The movement in the East-West direction is called Fast-Scan, and the movement in North-South direction is called Slow-scan (Fig. 2.6). The Scan area can be placed anywhere in the Field-of-Regard of the instrument. The FOR is $24 \mathrm{deg}(\mathrm{N}-\mathrm{S})$ and $18 \mathrm{deg}(\mathrm{E}-\mathrm{W})$. The start co-ordinates of the scan are defined by slow-scan and fastscan offset

The imaging is carried out by bi-directional scanning mechanism facilitated by controlled movement of scan-mirror in East-West and North-South directions. The coverage is wide $(\sim 18 \mathrm{degE}-\mathrm{W} \times 18 \mathrm{degN}-\mathrm{S})$ with repeativity of half-hour. Imaging is done in day as well as night time. The scan mirror operates in step and dwell mode to facilitate sounding. In East-West direction the mirror dwells at each step for $0.1,0.2$ or $0.4 \mathrm{sec}$ as per ground command. Within the scan mechanism, there is a provision to accept motion compensation signals from Attitude-Orbit Control System (AOCS) to compensate for various spacecraft rates, orbit perturbations and effect of the Imager mirror slew on the spacecraft. It improves the registration and navigation parameters of the image. GEOS projection is a navigation model computation based on the parking longitude alone for a virtual satellite without any requirement on s/c navigation data used for coarser geographic coordinates tagging on an idealized earth model, which is a perfect ellipsoid with an equator radius of $6378.1690 \mathrm{~km}$ and a polar radius of $6356.5838 \mathrm{~km}$. It provides a global and initial geometric reference without any ancillary data requirement. For INSAT-3D 
with unit in meter, the above projection cab be defined by following string " + proj=geos $+\mathrm{a}=6378137.0+\mathrm{b}=6356752.3142$ + lon_0 $=82.0+\mathrm{h}=35785831.0$ " in GDAL library for projection computations as shown in Fig.1 [3].
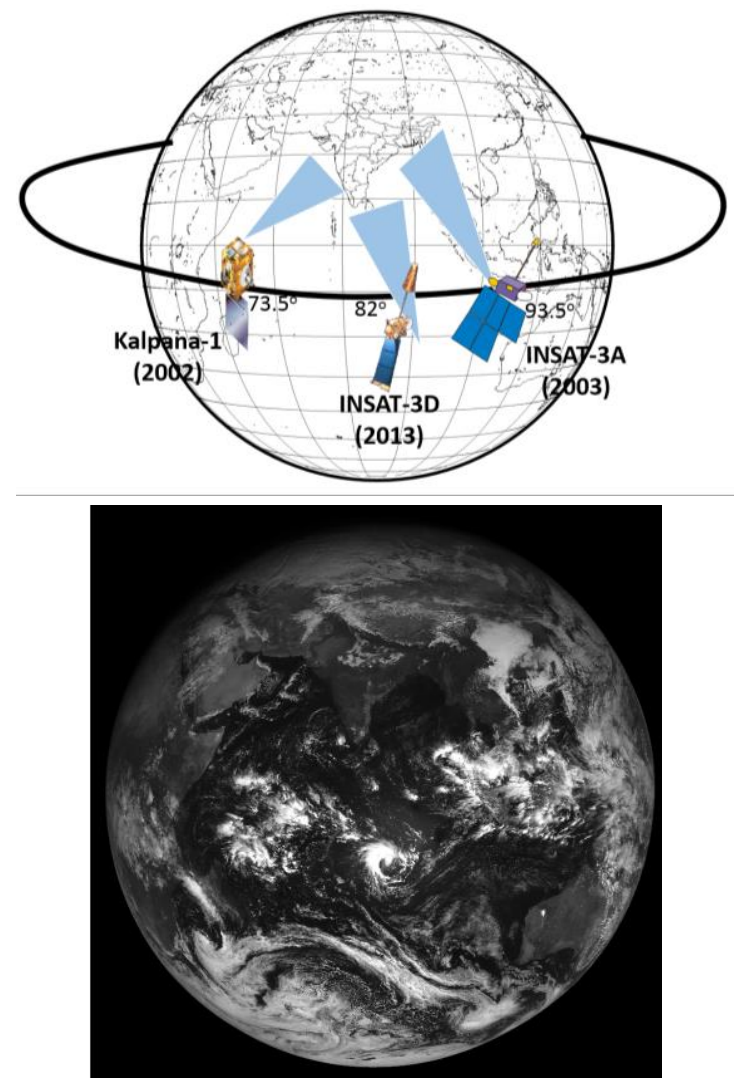

Fig.1. Few Indian Meteorology Platforms and INSAT-3D Visible Data 0600 UTC 10th January 2014

\subsection{DATA ACQUISITIONS, PROCESSING AND APPLICATIONS}

Data acquired by INSAT-3D is systematically corrected to generate basic products called as Level 1 products. Geo-physical parameters (Level 2 and Level 3) are generated by parameter retrieval algorithms using basic data products. Radiometric corrections include relativization, pixel-line loss correction, detector normalization and generation of calibration coefficients for Count to Radiance (for Visible) and Count to Brightness temperature conversion (for IR). Geometric corrections are carried out for servo correction, navigation and accurate feature registration between the successive half hourly images.

Geo-physical parameters such as Sea-Surface Temperature (SST), Upper Tropospheric Humidity (UTH), Water Vapour Wind (WVW), Out-going Long-wave Radiation (OLR) are operationally derived from INSAT-3D data and disseminated by IMD. Applications of INSAT-3D data include monitoring the growth of thunderstorms, tracking movements of tropical cyclones, monsoon depressions, onset and progress of monsoon systems and detailed atmospheric studies. The spectral bands and modes of IMAGER/SOUNDER are specially designed to permit frequent scanning to obtain understanding of dynamics of weather systems.

\subsection{IMAGE REGISTRATION REQUIREMENT}

INSAT-3D images obtained from imager are first required to be registered among themselves to carry forward for any scientific analysis and geophysical parameter retrievals. The emphasis on sub pixel accuracies in geometric fidelity is always found in the scientific literature as it is obvious and critical. The geometric fidelity requirements can't be undermined under any circumstances as the images considered here are acquired at a coarser resolution of about $1 \mathrm{~km}$ to $8 \mathrm{~km}$. And any mis-registration will show up directly and indirectly in derivations mentioned in the earlier paragraph. The Fig.2 shows the overlay of the land boundary data mismatch with INSAT-3D data covering left of Indian sub-continent before and after registration using a reference image.
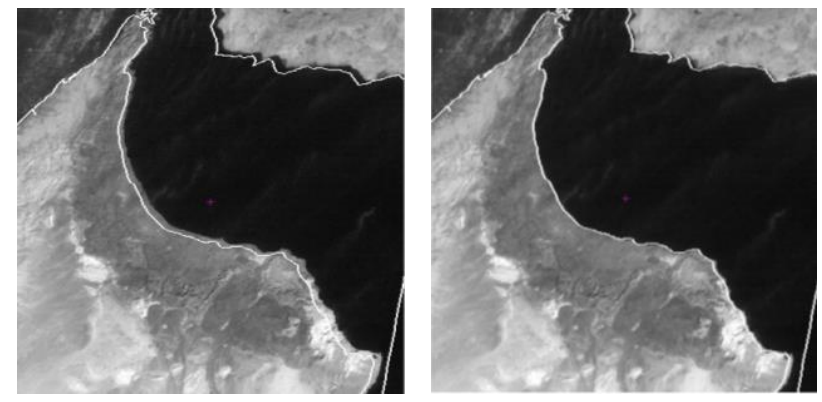

Fig.2. land water boundary overlay on INSAT-3D visible data

\section{CURRENT STATE OF PROBLEM RESOLUTION}

It is a very old and continuing problem to address registration of meteorological images and there are plenty of developments in this area of specialization as detailed here. Kovacs and Szcnyan outlined an approach in their work: Navigation models based only on using ground control points (GCPs) has a major disadvantage that the identification of such reference points is not possible in cloudy and open ocean regions [4]. Use of reference images and validation of orbital parameters is superior to the GCP based approach. This approach was used to register AVHRR images for applications related to surface radiation balance, temperature retrievals etc. Another model based approach experimented by Katamanov by using automatic selection of GCPs and attitude forecasting [5]. He claims to have achieved one-pixel level accuracy within a session of meteorology image acquisitions from satellites POES NOAA, MTSAT-1R and FengYun-2C with success rate $>95 \%$. Land ocean separability hypothesis was put to use by them to increase the reliability of GCP selection. The land-ocean separability test is tough in complex weather conditions according to Katamanov work. They could show a scheme based on consistent orbital and attitude parameter correction and the scheme may be used for navigation parameter forecasting of adjacent satellite passes also for FY-1D images. Verification of sea-land separability hypothesis for automatic ground control point generation can increase significantly the opportunity of image navigation under complex weather conditions of observation. They also reported that full FY-1D images cannot be navigated accurately by orbital and attitude parameter correction alone. 
Patt and Woodward [6] developed a method for SeaWiFS platform acquired ocean sensor data improve geometric accuracy to use island features as targets. In this approach, the acquired pixel in consideration was classified beforehand whether it is a land or ocean or cloud pixel. Land and water pixels' identification was extended to find island pixel clusters. The island pixel locations were compared to catalogued island data created by the authors themselves. Brunel and Marsouin [7] developed an image navigation model that combines a precise image deformation model (sensor imaging model) and an automatic adjustment on coastal landmarks. Authors were of the view that some of the required input parameters of the model are not known in real time with sufficient accuracy. In that case above mentioned method, provides a physical interpretation of image navigation errors and the results obtained on an image can be more easily used on another image.

Mao et al. [8] outlined an automatic approach for integrating images from multi temporal and multi sensor remote sensing based on coastlines derived satellite images using edge detection techniques. This technique was used to register SeaWiFS and AVHRR images using correlation-relaxation technique to find out correspondences between image points. They implemented a decision rule for grading the goodness of selected control points to estimate the transformation chosen. The relationship between the accuracy of registration and the number of GCPs indicates that a large number of GCPs will leads to more accurate image registration. The correctness of GCPs can also improve the accuracy of geometric registration. The approach can be used particularly well to register images of coastal areas. Cheng et al. proposed a new approach based on Affine Invariant Feature Matching (AIFM) with a filtering technique is proposed for automatic registration of remotely input image in coastal areas [9] [10]. The novelty of this approach was is an automatic filtering technique using RANdom SAmple Consensus (RANSAC) [11] with shoreline constraint for AIFM to remove all wrong matches and simultaneously keep as many correct matches as possible. To implement it, a progressive threshold strategy (from small value to large value) is presented to determine an appropriate RANSAC threshold, in which the progressive process is guided by shoreline constraint. The proposed approach (with filtering) is compared with standard AIFM (without filtering) using two typical image pairs in coastal areas. The experimental results indicate that the proposed approach can always provide much better matching results than standard AIFM for AVHRR image registration.

These techniques discussed above are more or less hybrid techniques, putting a navigational model, automatic collection of landmarks, or coast line segments, matching them, and most importantly employing a procedure to remove wrong matching, points and improve the robustness to register meteorological images with at least +/- one resolution unit distance. It is easy to see that geostationary imaging platform acquired images portray a big portion of earth with weatherly influences such as clouds are found commonly. Any automatic registration procedure need to handle these type of realities. Let us understand the feature based and intensity based approaches with few selected data experiments. Though the main aim of the paper is to see the performance accuracy of the intensity based image registration approach, a comparison is always expected to validate the approach. In comparison, level of sophistication of the techniques involved needs to be matched for a fair play. So therefore, efficient procedures are developed in both feature and intensity based approaches in this work. Let us start with the feature based approach developed for the above purpose.

\section{PROPOSED CONTOUR FEATURE BASED REGISTRATION APPROACH}

Automatic registration of MET images is a difficult problem to deal with as the feature collection from the low contrast, low SNR images are extremely error prone leads to many failures in image matching due to homogeneous patches present in the data. Simple approaches may not handle such complex issues as apparently seen in feature based registration. A clever choice could be to generate a contour map separating land and water not occulted by cloud. Such contour regions could be used for correlating features which are permanent and not transient as clouds or snow. As we have already seen that, INSAT-3D has SWIR and VIS channels and they could be used generate such contours.

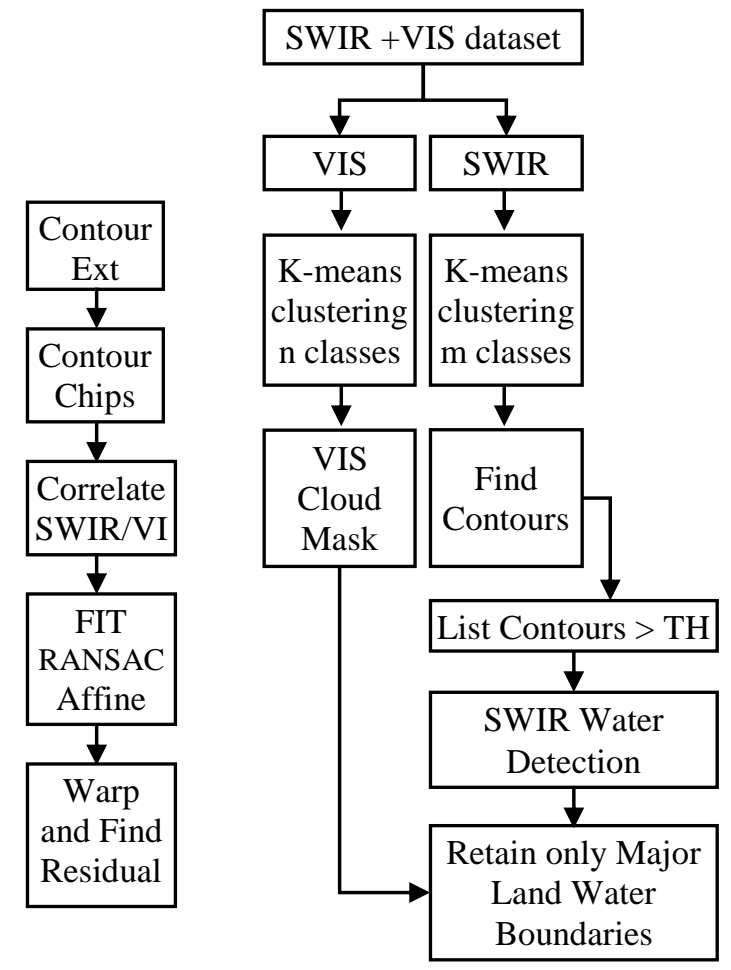

Fig.3. Contour block phase correlation based registration

\subsection{LAND, WATER AND CLOUD MASK GENERATION}

Single image based cloud detection is generally achieved by checking the gray value of the pixel is greater than a predefined threshold. Let us reminded that VIS channel can be used during daytime only, and SWIR type of channels can be used during day and night times. The problem with threshold based approach is that, they have to be dynamically found, and it is difficult for regions with coastal features. Better techniques can be employed for the detection using SWIR channels effectively to separate water pixels to a larger extent, and VIS can be used for detecting cloud features. Both of these can be done using a k-means data clustering techniques with far spread random clusters to group 
disjoint samples in to a definite number of clusters of equal variance using the sum of square minimising criteria within the cluster as the features targeted are well expressed in the data [12] - [14]. Merging smaller clusters to major ones and using VIS clusters over SWIR clusters in non-water regions. VIS/SWIR images were clustered with 10/7 classes originally and merging small classes into bigger one Contour detection and eliminating smaller contour regions can yield as a contour map. Within this contour map, continuous segments are captured for matching image portions. The complete steps in contour based image portion detection and matching in Fig. 3. However, a good number of image portions for matching shall be available and this can be achieved by thresholding the size of the contour area. The Fig. 4 depicts the extracted boundaries using $20^{\text {th }}$ Nov, 2017 and $10^{\text {th }}$ Jan, 2014 datasets. The top row shows the VIS and SWIR images themselves, and the second row shows the clustered data for both channels corresponding to cloud, snow and bright regions heighted and SWIR channel showing ocean pixels bright. The third row first column show the first land water separated boundary line segments and the second column showing the continuous segments in contours. The fourth row show the contiguous segments considered for phase correlations and the right column shows the sub regions marked over the data.

\subsection{IMAGE MATCHING BY PHASE CORRELATION}

Feature based matching uses normalised cross correlation in the spatial domain approach or phase correlation in the frequency domain approach. Phase correlation based image matching is employed here. Phase correlation method (PCM) is a Fourier domain method used for estimating translations between images in a robust way. It generates a phase difference map that contains a single peak. The location of the peak is found in proportion to the relative $2 \mathrm{D}$ shift between 2 images. The PCM is resilient to noise and image defects and is readily automated. It is completely equivalent to correlation in the spatial domain, but the calculation is orders of magnitude faster in the Fourier domain. Fourier Shift Theorem states if $f_{1}$ and $f_{2}$ are two images that differ only by a displacement $\left(\Delta_{x}, \Delta_{y}\right)$ such that,

$$
\begin{gathered}
f_{2}(x, y)=f_{1}\left(x-\Delta_{x}, y-\Delta_{y}\right) \\
F_{2}\left(\omega_{x}, \omega_{y}\right)=F_{1}\left(\omega_{x}, \omega_{y}\right) e^{-j\left(\omega_{x} \Delta_{x}+\omega_{y} \Delta_{y}\right)}
\end{gathered}
$$

The normalized cross power spectrum is given by

$$
C_{f 1 f 2}\left(\omega_{x}, \omega_{y}\right)=\frac{F_{1}\left(\omega_{x}, \omega_{y}\right) F_{2}^{*}\left(\omega_{x}, \omega_{y}\right)}{\left|F_{1}\left(\omega_{x}, \omega_{y}\right) F_{2}^{*}\left(\omega_{x}, \omega_{y}\right)\right|}=e^{j\left(\omega_{x} \Delta_{x}+\omega_{y} \Delta_{y}\right)}
$$

Inverse FFT of $C_{f 1 f 2}\left(\omega_{x}, \omega_{y}\right)$ results in a direct delta function having a single peak. Finding out maximum of this surface in both row and column directions and then arriving at a middle point in the 2D space, we can achieve sub-pixel level information in matching [15] - [20]. Finding the relative displacement

$$
\phi\left(\Delta_{x}, \Delta_{y}\right)=\sum_{\omega_{x}=-\pi}^{\pi} \sum_{\omega_{y}=-\pi}^{\pi} W\left(\omega_{x}, \omega_{y}\right)\left|C_{f 1 f 2}\left(\omega_{x}, \omega_{y}\right)-e^{j\left(\omega_{x} \Delta_{x}+\omega_{y} \Delta_{y}\right)}\right|^{2}
$$

where $W$ is the weight matrix and minimizes $\phi\left(\Delta_{x}, \Delta_{y}\right)$ to obtain $\left(\Delta_{x}, \Delta_{y}\right)$.
20 Nov 2017
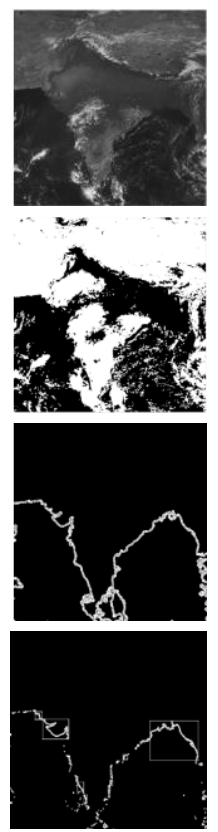
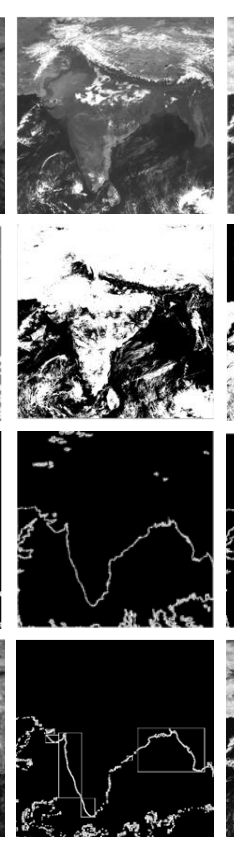

$10 \mathrm{Jan} 2014$

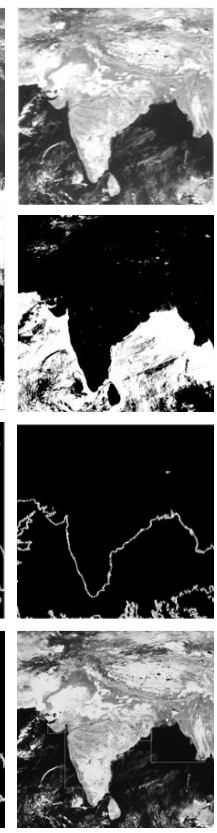

Fig.4. VIS/SWIR (Column 1 and 2) belongs to 20 Nov 2017 and VIS/SWIR (Column 3 and 4) belongs to 10 Jan 2014 acquisitions

\subsection{AFFINE USING RANSAC}

An affine model is built with predicted and phase correlated positions of the image at those contour boxes. A RANSAC procedure is employed to weed out outliers before estimating a final affine between the image pair. The residuals before and after shall be estimated to measure the performance [10] [11].

\section{PROPOSED INTENSITY REGISTRATION APPROACH}

BASED

In intensity based approach the alignment error is minimised through an optimizer framework. The alignment is improved progressively by guessing the direction and step size estimated using the derivatives of the image to image matching quality under the chosen transformation between them [21] - [25]. The initial transformation should be near the final solution for a robust estimation otherwise, few preparatory steps are to be executed such as setting up a multi resolution pyramid for hierarchical refinement of the registration model from coarse to fine scale of the data as represented in the Fig.5. Let us define a fixed image used as reference $A(x)$ and moving image $B(x)$ to be warped to the fixed image with a transformation choice $T(x, \mu)$ with $x$ as the image location and $\mu$ as the transformation parameter vector refined at every iterative step. The following minimization problem is considered:

$$
\hat{\mu}=\arg \min C(A, B \cdot T)
$$

where, $C$ is the cost function that measures the similarity of the fixed image and the deformed moving image. The solution $\hat{\mu}$ is the parameter vector that minimizes that cost function. To 
determine the optimal set of parameters, an iterative optimization strategy is employed as follows.

$$
\mu_{k+1}=\mu_{k}+a_{k} d_{k}, k=0,1,2, \ldots, N
$$

where $d_{k}$ is the search direction at iteration $k$ usually estimated from the gradient of the cost function and $a_{k}$ a scalar gain factor either set as a constant or slow decay function controlling the step size along the search direction per iteration basis. The search directions and gain factors are chosen such that the sequence $\mu_{k}$ converges to a local minimum of cost function $C$ using a gradient descent mechanics. The cost function $C$ mentioned in Eq.(2) is chosen as the negative value of mutual information $M(A, B)$ between two random variables $A$ and $B$ can be defined as

$$
M(A, B)=\sum_{a} \sum_{b} p_{A, B}(a, b) \log \frac{p_{A, B}(a, b)}{P_{A}(a) P_{B}(b)}
$$

It brings a measure between the joint $\operatorname{PDF} p_{A, B}(a, b)$ of two random variables $A$ and $B$ and the product of their marginal PDFs, $p_{A}(a) p_{B}(b)$, which is the measure of the match. The estimation of PDFs, MI, and its derivative are achieved by computing a stochastic joint histogram using Parzen windowed density estimate approach outlined in earlier works [22]. This improvises the whole estimate as a closed or analytical solution. The optimizer mechanics as mentioned earlier can be a standard gradient descent or an adaptive stepsize based. Then such an approach is termed as Adaptive and Stochastic Gradient Descent approach [24]. In such a scenario, every iteration different samples are collected randomly in the overlapping image region to compute joint histogram and other estimates to progressive update the transform parameters given in equation 6 using variation calculus steps.

\section{PERFORMANCE COMPARISON}

Feature and intensity approaches have been described is put to use to demonstrate the performance comparison of both the techniques. Data sets selected are VIS and SWIR channel sets. The Fig. 6 and Fig.7 depicts the results for images acquired at 0230 and 0530 UTC out of the 48 acquisitions in a day. The image shown in top left block is VIS data and right down below shown is the boundary data extracted using both the channel data, marked with correlatable blocks for feature based approach. Residual maps shown by three plots for input (before registration), using feature matching by phase correlation and the intensity based method advocated. The residuals obtained at 100 by 100 grid locations for creating pixel wise residuals by spatial binning of block size 64. Input shows more residuals at bottom locations which include some cloudy patches over ocean and land portions as well. The same regions in feature based method show lesser residuals and in intensity based approach it is further reduced indicating that, intensity based method scoring better in registration of these data sets. The scatter plots shown in a row also belongs to input, feature, and intensity registered sets, showing reducing range of residuals. The mean and standard deviation of the residuals are tabulated also (Table.3, Table.4 and Table.5 and Fig.8), after outliers rejected beyond a range of $+/-5$ pixels with $50 \times 50$ correlations. Residual maps give us better insights than scatter plots and table data due to outlier rejection criteria and computing statistics for a single performance value.

Multiresolution image pair

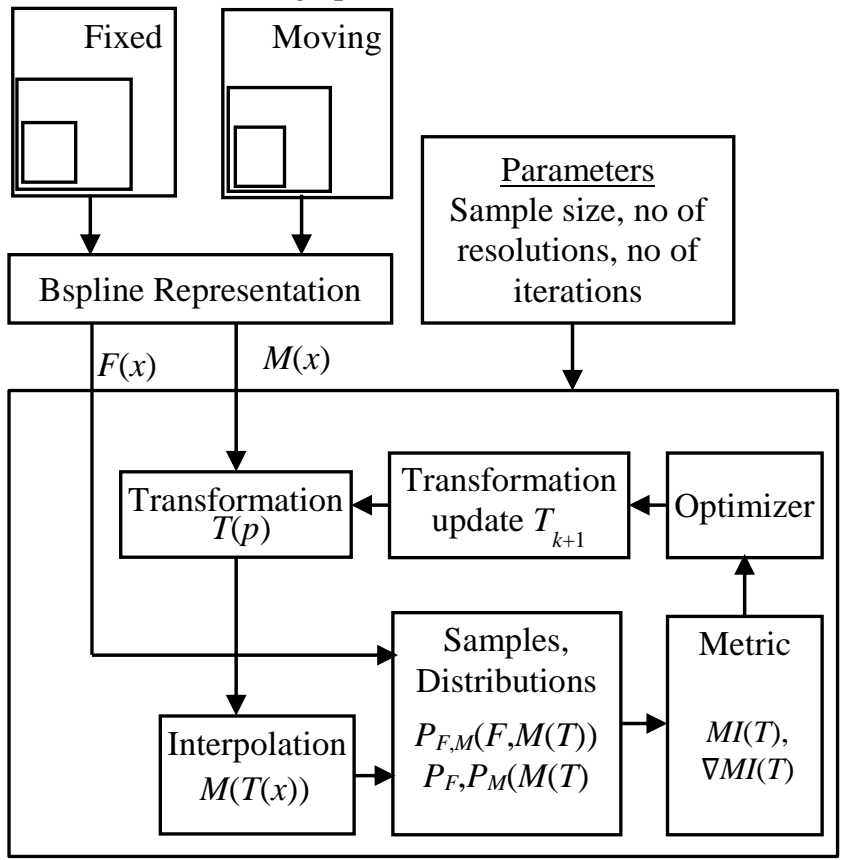

Fig.5. Intensity based MET image registration scheme

\section{UTC}
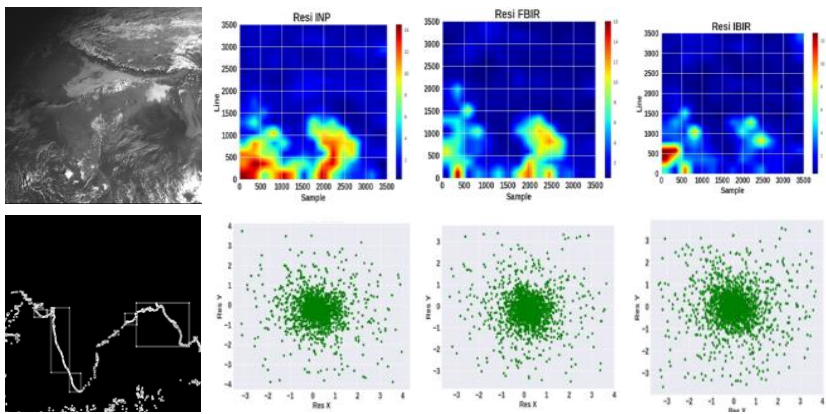

Fig.6. 10th Jan, 20140230 UTC VIS Image, boundary data extracted, Residual Map, and Scatter plots

\section{UTC}
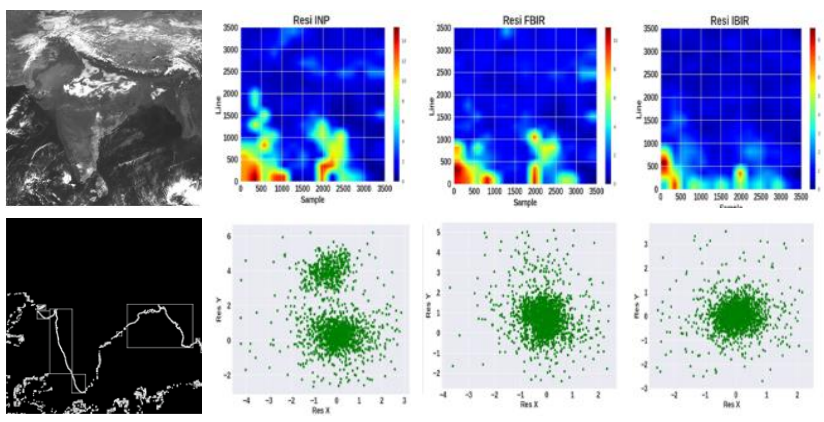

Fig.7. 10 $0^{\text {th }}$ Jan, 20140530 UTC VIS Image, boundary data extracted, Residual Map, and Scatter plots 


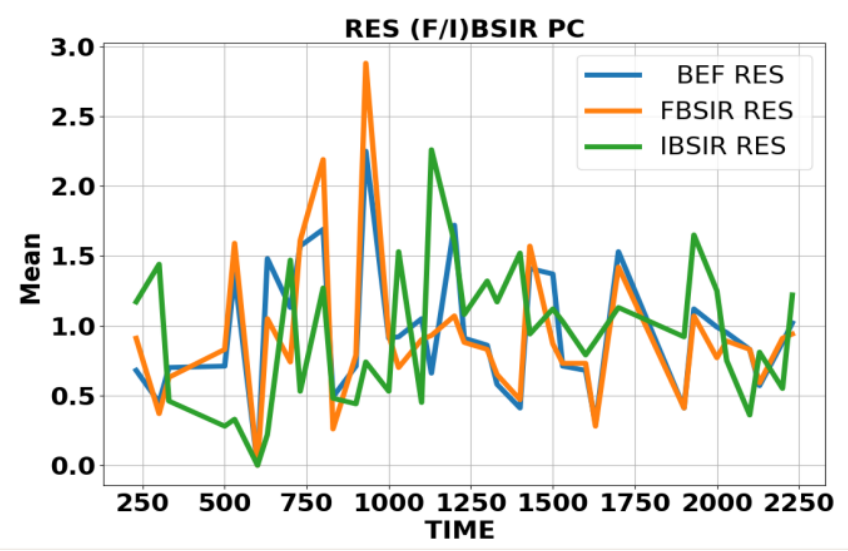

Fig.8. Mean Residuals by feature and intensity registrations

Table.3. Residuals estimated for VIS channel before registration

\begin{tabular}{|c|c|c|c|c|c|}
\hline \multirow{2}{*}{ Time } & \multicolumn{2}{|c|}{ Residual } & \multirow{2}{*}{ Time } & \multicolumn{2}{c|}{ Residual } \\
\cline { 2 - 3 } \cline { 5 - 6 } & Mean & Std & & Mean & Std \\
\hline 0230 & 0.68 & 0.01 & 1230 & 0.91 & 0.3 \\
\hline 0300 & 0.45 & 0.16 & 1300 & 0.86 & 0.23 \\
\hline 0330 & 0.7 & 0.19 & 1330 & 0.58 & 0.22 \\
\hline 0500 & 0.71 & 0.19 & 1400 & 0.41 & 0.03 \\
\hline 0530 & 1.37 & 0.33 & 1430 & 1.41 & 0.04 \\
\hline 0600 & 0.0 & 0.0 & 1500 & 1.37 & 0.18 \\
\hline 0630 & 1.48 & 0.24 & 1530 & 0.71 & 0.11 \\
\hline 0700 & 1.13 & 0.71 & 1600 & 0.68 & 0.07 \\
\hline 0730 & 1.57 & 1.02 & 1630 & 0.3 & 0.14 \\
\hline 0800 & 1.69 & 0.24 & 1700 & 1.53 & 0.53 \\
\hline 0830 & 0.49 & 0.03 & 1900 & 0.41 & 0.16 \\
\hline 0900 & 0.71 & 0.11 & 1930 & 1.12 & 0.42 \\
\hline 0930 & 2.25 & 0.23 & 2000 & 0.99 & 0.16 \\
\hline 1000 & 0.91 & 0.01 & 2030 & 0.95 & 0.39 \\
\hline 1030 & 0.92 & 0.12 & 2100 & 0.83 & 0.22 \\
\hline 1100 & 1.05 & 0.39 & 2130 & 0.57 & 0.25 \\
\hline 1130 & 0.66 & 0.11 & 2200 & 0.88 & 0.44 \\
\hline 1200 & 1.72 & 0.66 & 2230 & 1.02 & 0.65 \\
\hline
\end{tabular}

Table.4. Residuals estimated for VIS data by feature based

\begin{tabular}{|c|c|c|c|c|c|}
\hline \multirow{2}{*}{ Time } & \multicolumn{2}{|c|}{ Residual } & \multirow{2}{*}{ Time } & \multicolumn{2}{c|}{ Residual } \\
\cline { 2 - 3 } \cline { 5 - 6 } & Mean & Std & & Mean & Std \\
\hline 0230 & 0.91 & 0.12 & 1230 & 0.88 & 0.13 \\
\hline 0300 & 0.37 & 0.27 & 1300 & 0.83 & 0.25 \\
\hline 0330 & 0.63 & 0.24 & 1330 & 0.65 & 0.35 \\
\hline 0500 & 0.83 & 0.32 & 1400 & 0.47 & 0.01 \\
\hline 0530 & 1.59 & 0.46 & 1430 & 1.57 & 0.12 \\
\hline 0600 & 0.0 & 0.0 & 1500 & 0.87 & 0.07 \\
\hline 0630 & 1.05 & 0.09 & 1530 & 0.73 & 0.14 \\
\hline 0700 & 0.74 & 0.15 & 1600 & 0.73 & 0.05 \\
\hline 0730 & 1.61 & 0.5 & 1630 & 0.28 & 0.17 \\
\hline
\end{tabular}

\begin{tabular}{|c|c|c|c|c|c|}
\hline 0800 & 2.19 & 0.39 & 1700 & 1.42 & 0.56 \\
\hline 0830 & 0.26 & 0.12 & 1900 & 0.41 & 0.16 \\
\hline 0900 & 0.79 & 0.42 & 1930 & 1.07 & 0.28 \\
\hline 0930 & 2.88 & 0.48 & 2000 & 0.77 & 0.16 \\
\hline 1000 & 0.93 & 0.04 & 2030 & 0.89 & 0.34 \\
\hline 1030 & 0.7 & 0.24 & 2100 & 0.83 & 0.22 \\
\hline 1100 & 0.9 & 0.02 & 2130 & 0.59 & 0.31 \\
\hline 1130 & 0.93 & 0.37 & 2200 & 0.91 & 0.34 \\
\hline 1200 & 1.07 & 0.11 & 2230 & 0.94 & 0.68 \\
\hline
\end{tabular}

Table.5. Residuals estimated for VIS data by intensity based

\begin{tabular}{|c|c|c|c|c|c|}
\hline \multirow{2}{*}{ Time } & \multicolumn{2}{|c|}{ Residual } & \multirow{2}{*}{ Time } & \multicolumn{2}{c|}{ Residual } \\
\cline { 5 - 6 } & Mean & Std & & Mean & Std \\
\hline 0230 & 1.17 & 0.44 & 1230 & 1.08 & 0.54 \\
\hline 0300 & 1.44 & 0.83 & 1300 & 1.32 & 0.85 \\
\hline 0330 & 0.46 & 0.23 & 1330 & 1.17 & 0.35 \\
\hline 0500 & 0.28 & 0.2 & 1400 & 1.52 & 0.45 \\
\hline 0530 & 0.33 & 0.12 & 1430 & 0.94 & 0.62 \\
\hline 0600 & 0.0 & 0.0 & 1500 & 1.12 & 0.7 \\
\hline 0630 & 0.22 & 0.06 & 1530 & 1.03 & 0.38 \\
\hline 0700 & 1.47 & 0.54 & 1600 & 0.79 & 0.07 \\
\hline 0730 & 0.53 & 0.01 & 1630 & 0.89 & 0.79 \\
\hline 0800 & 1.27 & 0.27 & 1700 & 1.13 & 0.72 \\
\hline 0830 & 0.48 & 0.22 & 1900 & 0.92 & 0.53 \\
\hline 0900 & 0.44 & 0.04 & 1930 & 1.65 & 0.97 \\
\hline 0930 & 0.74 & 0.16 & 2000 & 1.25 & 0.51 \\
\hline 1000 & 0.53 & 0.13 & 2030 & 0.75 & 0.49 \\
\hline 1030 & 1.53 & 0.55 & 2100 & 0.36 & 0.3 \\
\hline 1100 & 0.45 & 0.4 & 2130 & 0.81 & 0.01 \\
\hline 1130 & 2.26 & 0.43 & 2200 & 0.55 & 0.02 \\
\hline 1200 & 1.6 & 0.18 & 2230 & 1.22 & 0.84 \\
\hline
\end{tabular}

\section{ATMOSPHERIC MOTION VECTORS DERIVATION}

Atmospheric Motion Vectors (AMVs) are the satellite derived winds, extracted from satellite imagery by tracking tracers such as clouds and water vapor through a series of consecutive satellite images. AMV includes cloud motion vectors and water vapor winds [26][27]. The validation of retrieved AMV is feasible by comparing to radiosonde network data analysis and calculating Mean Vector Difference (MVD), Root Mean Square vector difference (RMSVD), and BIAS. Our registration scheme agrees well with the AMV derived from METEOSAT-7 CIMSS data sets. 


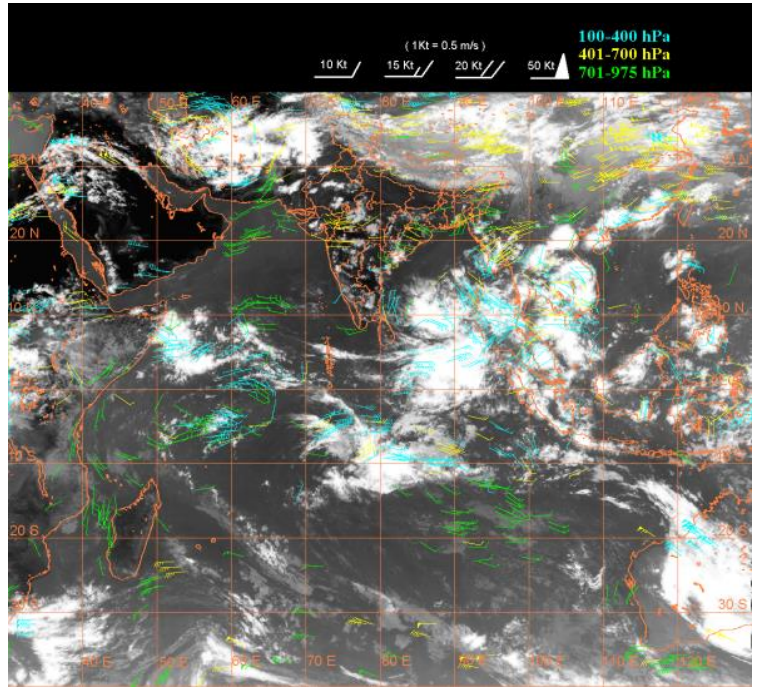

Fig.9. AMV derived with intensity based registration for TIR1 $16^{\text {th }}$ May, 20141200 UTC

Table.6. AMV retrieved at 00 and 12 UTC comparison

\begin{tabular}{|c|c|c|c|c|c|c|}
\hline \multirow{2}{*}{ PAR-AM } & \multicolumn{3}{|c|}{ INSAT-3D registered } & \multicolumn{3}{|c|}{ Meteosat-7: CIMSS } \\
\cline { 2 - 7 } & HIGH & MID & LOW & HIGH & MID & LOW \\
\hline MVD & 4.71 & 4.64 & 3.96 & 4.53 & 4.25 & 4.19 \\
\hline RMSVD & 5.37 & 5.14 & 4.30 & 5.30 & 4.77 & 4.49 \\
\hline SD & 2.48 & 1.94 & 1.36 & 2.87 & 2.02 & 1.36 \\
\hline BIAS & -0.17 & -.22 & 1.22 & -0.51 & -0.61 & 0.22 \\
\hline NC & 1907 & 487 & 222 & 3276 & 457 & 402 \\
\hline
\end{tabular}

*HIGH (100-400) hPa; MID (401-700) hPa; LOW (701-975) hPa

INSAT-3D derived AMV and from METEOSAT-7 CIMSS data sets agree by evaluating MVD, RMSVD, SD. It testifies that, the registration accuracy is at reasonable level, the differences are found owing to the fact that, radiometry and the geometry of the data are different for different imaging platform. We will not further elaborate on the AMV derived as our main focus is kept on the registration scheme in this work. This exercise was an external exercise to show final use of the registered set and not included as a main contribution.

\section{CONCLUSIONS}

We could demonstrate two state of art algorithms belonging to two different methodologies with feature detection and matching and the other an intensity based one without any feature detection step for registering MET images acquired by INSAT-3D VIS, SWIR channels. In both the methods, navigation details were not used for preparing the images for registration. Contour feature based methods supposed to have a slight edge over the intensity based approach in these data sets as they exhibit low contrast, lower SNR and cloud, snow occlusions on the first scrutiny. However, both approaches are comparable for sub pixel accuracies. The boundary extraction using single images was not attempted in this effort, but it is not very difficult to include such a procedure either.

\section{ACKNOWLEDGMENTS}

The authors thank Shri. Tapan Misra, Director, Space Applications Centre, ISRO, Ahmedabad who supports us in every scientific endeavor objectively, and Shri. Debjyoti Dhar, Group Director, SIPG, SAC, for his encouragement and S.K. Deb our colleague for his help. We thank SRM Institute of Science and Technology for academic support in research activities.

\section{REFERENCES}

[1] Indian Space Research Organisation, "INSAT-3D", Available at: http://www.isro.gov.in/Spacecraft/INSAT-3DISRO.html, Accessed on 2016.

[2] N. Padmanabhan, R. Ramakrishnan and S.B. Gurjar, "Geometric modelling of INSAT-2E CCD Payload and Multi Strip Mosaicking for Geocoding Large Areas", Current Science, Vol. 86, No. 8, pp. 1113-1121,2004.

[3] "GDAL: OGR Projections Tutorial", Available at: http://www.gdal.org/osr_tutorial.html, Accessed on 2018.

[4] L. Kovacs and I.G. Szcnyan, "Development of AVHRR Image Registration in Hungary", Advanced Space Research, Vol. 17, No. 1, pp. 123-126, 1996.

[5] S.N. Katamanov, "Automatic Navigation of One Pixel Accuracy for Meteorological Satellite Imagery", Proceedings of $1^{\text {st }}$ Russia and Pacific Conference on Computer Technology and Applications, pp. 269-274, 2010.

[6] F.S. Patt and R.H. Woodward, "An Automated Method for Navigation Assessment for Earth Survey Sensors using Island Targets", International Journal of Remote Sensing, Vol. 18, No. 16, pp. 3311-3336,1997.

[7] P. Brunel and A. Marsouin, "Operational AVHRR Navigation Results", International Journal of Remote Sensing, Vol. 21, No. 5, pp. 951-972, 2000.

[8] Z. Mao, D. Pan, H. Huang and W. Huang, "Automatic Registration of SeaWiFS and AVHRR Imagery", International Journal of Remote Sensing, Vol. 22, No. 9, pp. 1725-1735, 2001.

[9] L. Cheng, L. Tong, Y. Liu, M. Li and J. Wang, "Automatic Registration of Coastal Remotely Sensed Imagery by Affine Invariant Feature Matching with Shoreline Constraint", Marine Geodesy, Vol. 37, No. 1, pp. 32-46, 2014.

[10] L. Cheng, Y. Pian, Z. Chen, P. Jiang, Y. Liu, G. Chen, P. Du and M. Li, "Hierarchical Filtering Strategy for Registration of Remote Sensing Images of Coral Reefs", IEEE Journal of Selected Topics in Applied Earth Observation and Remote Sensing, Vol. 9, No. 7, pp. 3304-3314, 2016.

[11] M.A. Fischler and R.C. Bolles, "Random Sample Consensus: A Paradigm for Model Fitting with Applications to Image Analysis and Automated Cartography", Communications of the ACM, Vol. 24, No. 6, pp. 381-395, 1981.

[12] R.O. Duda, P.E. Hart and D.G. Stork, "Pattern Classification", $2^{\text {nd }}$ Edition, Wiley, 2000.

[13] S. Eken and A. Sayar, "An Automated Technique to Determine Spatio-Temporal Changes in Satellite Island Images with Vectorization and Spatial Queries", Sadhana, Vol. 40, No. 1, pp. 121-137, 2015.

[14] L. Sun, X. Mi, J. Wei, J. Wang, X. Tian, H. Yu and P. Gan, "A Cloud Detection Algorithm-Generating Method for 
Remote Sensing Data at Visible to Short-Wave Infrared Wavelengths", ISPRS Journal of Photogrammetry and Remote Sensing, Vol. 124, pp. 70-88, 2017.

[15] H. Stone, M. Orchard and E.C. Chang, "Subpixel Registration of Images", Proceedings of $33^{\text {rd }}$ Asilomar Conference on Signals, Systems, and Computers, pp. 14461452, 1999.

[16] W.S. Hoge, "A Subspace Identification Extension to the Phase Correlation Method", IEEE Transactions on Medical Imaging, Vol. 22 No. 2, pp. 277-280, 2003.

[17] F. Homblot, B. Collin and A. Mohammad-Djafari, "Evaluation and Practical Issues of Image Registration using Phase Correlation Methods," Proceedings of International Conference on Physics in Signal and Image Processing, pp. 113-118, 2005.

[18] J. Ren, T. Vlachos and J. Jiang, "Subspace Extension to Phase Correlation Approach for Fast Image Registration", Proceedings of International Conference on Image Processing, pp. 481-484. 2007.

[19] M. Guizar-Sicairos, S.T. Thurman and J.R. Fienup, "Efficient Subpixel Image Registration Algorithms", Optics Letters, Vol. 33, No. 2, pp. 156-158, 2008.

[20] S. Cao, J. Jiang, G. Zhang and Y. Yuan, "An Edge-based Scale-and Affine-Invariant Algorithm for Remote Sensing Image Registration", International Journal of Remote Sensing, Vol. 34, No. 7, pp. 2301-2326, 2013.

[21] F. Maes, A. Collignon, D. Vandermeulen, G. Marchal and P. Suetens, "Multimodality Image Registration by
Maximization of Mutual Information", IEEE Transactions on Medical Imaging, Vol. 16, No. 2, pp. 187-198, 1997.

[22] P. Thevenaz and M. Unser, "Optimization of Mutual Information for Multiresolution Image Registration", IEEE Transactions on Image Processing, Vol. 9, No. 12, pp. $2083-$ 2099, 2000.

[23] A.A. Cole-Rhodes, K.L. Johnson, J. Le Moigne and I. Zavorin, "Multiresolution Registration of Remote Sensing imagery by Optimization of Mutual Information using a Stochastic Gradient", IEEE Transactions on Image Processing, Vol. 12, No. 12, pp. 1495-1511, 2003.

[24] S. Klein, J.P.W. Pluim, M. Staring and M.A. Viergever, "Adaptive Stochastic Gradient Descent Optimisation for Image Registration", International Journal of Computer Vision, Vol. 81, No. 3, pp. 227-239, 2009.

[25] S. Klein, M. Staring, K. Murphy, M.A. Viergever and J.P.W. Pluim, "Elastix: A Toolbox for Intensity-Based Medical Image Registration", IEEE Transactions on Medical Imaging, Vol. 29, No. 1, pp. 196-205,2010.

[26] C.M. Kishtawal, S.K. Deb, P.K. Pal and P.C. Joshi, "Estimation of Atmospheric Motion Vectors from Kalpana1 Imagers", Journal of Applied Meteorology and Climatology, Vol. 48, No. 11, pp. 2410-2421, 2009.

[27] S.K. Deb, C.M. Kishtawal, P.K. Pal and P.C. Joshi, "A Modified Tracers Selection and Tracking Procedure to Derive Winds using Water Vapor Imagers", Journal of Applied Meteorology and Climate, Vol. 47, No. 12, pp. 3252-3263, 2008. 